\title{
A game theory approach with dynamic pricing to optimize smart grid operation
}

\author{
Makhlouf Hadji ${ }^{\mathrm{a}}$, Marc Girod-Genet ${ }^{\mathrm{b}}$, Hossam Affifi ${ }^{\mathrm{b}^{*}}$ \\ ${ }^{a}$ Technological Research Institute - IRT SystemX,8 avenue de la vauve, 91120, Palaiseau, France \\ ${ }^{b}$ Institut Mines-Telecom, telecom SudParis, 17 rue charles fourier, 91010, Evry, France
}

\begin{abstract}
Smart Grids components include scalable metering, energy prediction (both production and consumption) and pricing. One of their goals consists to attract consumers to use green energy, to promote periods of low consumption and to dissuade customers from using their greedy devices during peak periods. The objective consists to determine the optimal suggested prices by the energy operator and the optimal demands of consumers. In this paper, we propose a theoretical model based on Stackelberg game to adjust prices of green energy. The proposed game is composed by a leader represented by the operator, and multiple followers represented by consumers. A Nash/Stackelberg equilibrium solution is found. Performance results confirm the uniqueness of Nash equilibrium and that a "best reply" dynamics for the repeated game converges to this equilibrium.
\end{abstract}

Keywords: Smart grids, game theory, optimization, dynamic pricing

\section{Introduction}

To meet the future power demand and the aim to reduce $\mathrm{CO}_{2}$ emissions designers of the next generation of electric power, distribution grid initiate a large research and technological action under the "Smart Grid" banner that starts to tackle some of the following issues:

- Significant reductions in residential peak demand energy consumption achieved by providing real-time price and environmental signals in conjunction with advanced in-home technologies.

- Integration of the green energy production from both second tier operators and private clients to reduce carbon footprint. This energy source is hence taking a major role in the future Smart Grid. Electric vehicles are also considered as very important future elements as they can affect the consumption peaks but they could also act as energy buffers to provide missing energy during these peaks [1].

- Provide an open infrastructure for newcomers to be easily integrated, in the same way as mobile telecom market has been deregulated.

- Provision of real time measurement and control tools that provide scalable and preferment actions on the energy grid.

The resulting architecture of a Smart Grid is defined as a standard power grid (traditional power distribution network) that is coupled with both a telecommunication network and a distributed information management system, and associated with services (handled within a energy-dedicated service architecture) to allow the following:

- Better energy consumption management,

- Better management of energy production and delivery (production versus consumption rationalization),

- Efficient and dynamic mix of heterogeneous energy sources (including renewable ones),

- Power outage impacts reduction,

*Manuscript received May 8, 2015; revised August 12, 2015.

Corresponding author. Tel.: +0-33-016-908-0630; E-mail address: makhlouf.hadji@irt-systemx.fr.

doi: $10.12720 /$ sgce.4.3.186-198 
- Dynamic pricing,

- Global energy efficiency increasing.

The telecommunication network is mainly used for metering/monitoring data collection and for the data distribution related to the enforcement of Smart Grid and energy management/control policies. The information management system is mainly carried out for the distributed storage and processing of data used for the decision making related to the Smart Grid and energy control/management operations. This Smart Grid architecture is therefore based on several and correlated key components (Real-time hybrid smart metering, distributed management and control infrastructure, data mining, data aggregation and data analysis infrastructure helping the actors to process and infer energy information and prediction and pricing tools), as shown in Fig. 1.

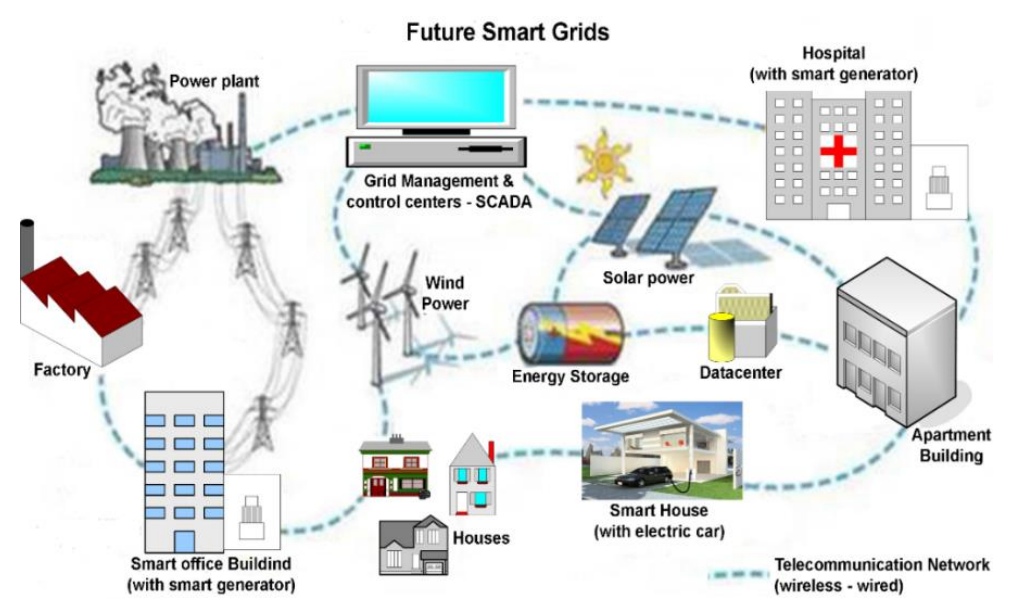

Fig. 1. Smart grid global view (GridWise Alliance).

An important element of the smart grid chain concerns the pricing. We believe that dynamic pricing is the only way to attract consumers to periods of low consumption and to dissuade them from using their greedy devices in peak periods. It is clearly the objective of this paper.

The main objective of dynamic pricing, at least from the energy provider side, is the adjustment of energy price according to market demand, e.g. increasing the price during peak periods. The expected effect is the customer shifting of its consumption to off-peak periods (cascading power outage avoidance). From the customer side, the main criteria that influences his consumption is the energy price (generally higher during rush hours i.e. peaks), but other criteria could be considered such as, for example, energy type (renewable or not) and customer profiles.

In our work, we consider the problem of constrained dynamic pricing in a Stackelberg/Nash manner taking into account finite resources or energy (limited resources) constraints. We also consider differentiated pricing proposals to better explore the revenue space and then select efficient strategies leading to higher payoffs. This differentiation can also be justified by considering clients in different geographic regions. Thus, we investigate game theoretical approaches to optimally determine the dynamic pricing menu to be proposed by the energy operator to customers. In the same time, we seek solutions to find optimal clients (often confused with customers) consumption demands according to the proposed prices.

The central goal of this paper is to find an agreement between customers and the operator. This allows different customers to partake in the interaction by proposing their respective demands and this in turn enable the energy operator to better manage its resources (energy). This is a non-cooperative game, where each customer is interested in maximizing her/his utility that can be modeled as a Stackelberg game [2], where the operator set prices and clients update their consumption to maximize their payoffs.

The remainder of this paper is organized as follows: Section II discusses a state of the art of game theory and the different components involved in a smart grid topology. Section III is dedicated to game 
theory approaches to handle with dynamic pricing problem. Section IV shows convergence steps to determine a Nash Equilibrium Point (NEP) of a game. Section V is dedicated to solve the one leader multiple followers' game. The objective consists to determine the optimal pricing proposal under some constraints. Conclusions and future work follow in Section VI.

\section{Related Work and Motivation}

Smart Grid key components has to be considered globally and the communication part should not be dealt with independently. Network is clearly not the main objective in the smart grid forums and standards, but it is a strong enabler. We should hence consider this as a use case with specific needs to propose adequate solutions and protocols. In this paper, we focus on one potential problem related to pricing. It cannot be achieved without the presence of a real-time and secure network infrastructure.

Intelligence, learning, processing, inference and self-adaptation have to be introduced in the power managing units and the control systems in order to make them cognitive, in every power grid level, processes and entities [3], [4]. This implies the support of decentralized and distributed control operations of the Smart Grid, as well as the carrying out of distributed and autonomous multi-criteria decision making. This also implies the design of a semantic open data model dedicated to the description and the inference of energy information/system knowledge. Such a knowledge model should in particular enable: the automation of control and monitoring systems, the better estimate of energy consumption levels, as well as the interworking of energy systems [5].

Smart Grids decision making related to pricing mainly rely: on the history modeling and prevision of both the energy production level and the energy consumption, as well as on the energy type (e.g. renewable or not) and price. For the client side (the consumer), we developed a tool in the VELCRI project [1] that learns his/her power consumption and that estimates the life of his batteries. It can predict the time remaining and can decide if the recharging process can wait for the low price energy period. The power provider is also learning the behavior of his clients on a minute/hour/day basis from one side and making prediction on the green power generation according to weather forecast on the other. He combines the two sets and produces power expectation per zone. This information is decisive to announce the pricing for the energy in high and low consumption periods (see Fig. 2).

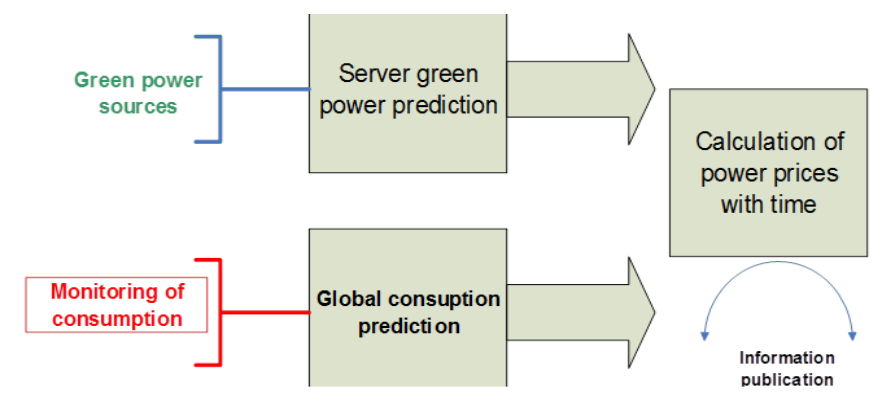

Fig. 2. Prediction scheme.

One can cite the U.S as an example of important pioneer country for the deployment of Smart Grids technologies and various forms of dynamic pricing already been considered over there [6] - [8];

- Time of Use (ToU), standard two part and pre-establish rate comprising on-peak and off-peak periods,

- Critical Peak Pricing (CPP), similar to ToU with a more expensive pricing strategy during peak and day hours. Generally, the estimate of next day load (peaks) trigger the switching of pricing strategies to $\mathrm{CPP}$,

- Critical Peak Rebates (CPR), comprises same critical pricing periods as CPP but provides customer with price rebates proportional to their consumption reduction during peaks,

- Real Time Pricing (RTP or Hourly Pricing - HP), offers prices reflecting the wholesale energy cost and varying according to an hourly basis, 
- Fixed Rate Pricing (FRP).

All these pricing strategies provide options for consumers to influence their electricity bill and have therefore to be considered in this paper.

In [8], the authors have developed 4 time-based pricing strategies (ToU, CPP, Peak Time Rebate or PTR and RTP). They have applied those pricing strategies to three consumer classes (Residential, Small and Medium General Service), their goal being to simulate the consumer responses for modeling the impact of their pricing strategies. In [7]-[9], authors show that, among all those pricing strategies, CPP was the most efficient in terms of peak reduction (up to $20 \%$ of energy usage reduction), while RTP enable a significant reduction of customer annual electricity bill (an average of $12 \%$ reduction).

Few studies have already been conducted for applying game theory to energy demand management and dynamic pricing. In [10], the authors have proposed a distributed strategy for energy demand management among multiple collaborating consumers and so they are not addressing the pricing problematic. In [11] and [12], the authors propose a multi-consumer dynamic pricing game using two strategies: opportunistic electricity or traditional one. In [11], the authors are only considering the scenario where one power source or generator (not the energy provider itself) is shared among multiple consumers. They only implement a simple pricing strategy based on client cooperation and message exchange. In [12], the authors try to incorporate multiple providers ( 2 types, renewable or not) into their game formulation. However, they both (i.e. [11], [12]) use a less dynamic consumer behavior than the one we are using and they both are not implementing the same utility functions as us.

In this paper, we propose a novel algorithm based on game theory principles to adjust the price and the demand using a game. The actors of this game are the energy provider (or for simplicity the operator) and the consumers. We consider a situation where each player can change its strategy one after the other and adopts a "best reply" response. It is shown that this dynamic converges to the Nash equilibrium [13] of the strategic game. Assuming the operator knows the consumer's utility function, this equilibrium point may give him some information to adjust its price leading to a desired level of consumption.

The game theory has been applied to model several information systems and communication problems. It can bring an innovative solution to systems with different independent players, where the action of each player has an incidence on the overall utility of the system [14]-[16] and [17].

In our context and after the abstraction of the network part, players correspond to energy providers and consumers. We may go beyond this first simple classification by introducing more players in the network side (infrastructure operator, mobile operator, facility operator, clearing house, etc...) but we restrict our analysis in this paper to a simple model. Note that in the simple model presented here, we may consider that the consumer represents in fact a set of several clients, all having the same utility function (and taking their action simultaneously).

Note also that the case where end client is buying and selling back energy when she generates green power, it is assimilated to the simple provider and client game.

The strategy chosen by players may be in general concurrent or cooperative. It is concurrent when the players compete on the resource and it becomes cooperative when players work together to improve the utility outcome.

In the Smart Grid context, we can find both cases. For example, identical energy operators may be concurrent to attract a client and they may be cooperative to overcome a peak energy consumption period.

\section{Dynamic Pricing Game Theory Approach}

We describe hereafter the solution we propose to optimize the system and to find an equilibrium point. We consider in the smart grid case in general and in this study in particular that the strategy of players is restricted to the production and the consumption of energy for both types of players (energy operators and end clients).

The best strategy for players in the smart grid context can be seen from two different perspectives.

The first best strategy could be a solution that minimizes the overall non green energy consumption. This could correspond to a patriotic action in favor of the planet, etc. 
The second strategy would be to maximize gains of energy operators and minimize expenditures of end clients.

In the first case, the pure strategies would correspond to the energy production and to the energy consumption actions.

We know from field trials that green energy can be easily lost because not consumed [18]. So we need to match the production with the expected consumption. While in conventional energy production phase adjustments can be made according to the environmental parameters such as time of day, of year, weather, etc.

Our algorithm is combining both approaches. We try to find the dynamic pricing strategy that improves green energy consumption and that is pricewise interesting for end customers.

The game tree can be sketched in Fig. 3 to understand the trends.

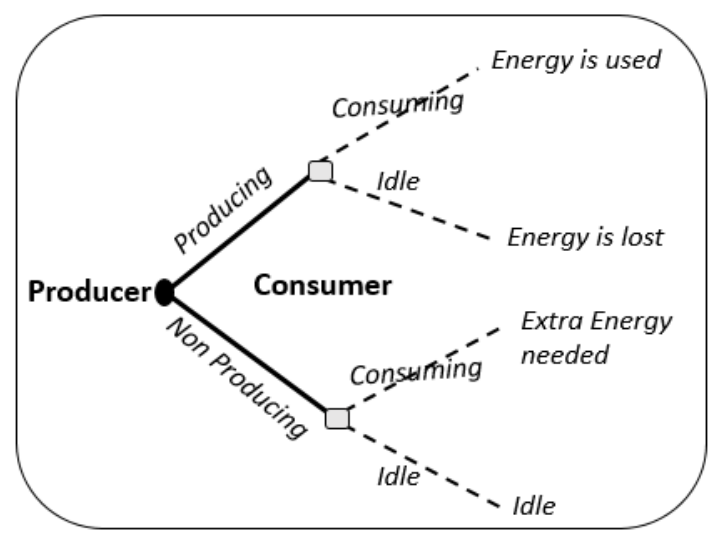

Fig. 3. The different cases in a smart grid simple context.

Fig. 3 shows essentially green energy production as a sequential game between energy operator with a vector of strategies given by (producing; non-producing), and customers with a strategy vector given by (consuming; idle). When the operator strategy consists to produce energy, the clients may consume it or not. In case of clients play "idle" strategy, then the energy produced is lost and this is a budget deficit for the operator, but in the same time, it is also considered as an amount of money not saved by clients if they consume later non green and more expensive energy. We derive the following normal form of the corresponding strategic game (i.e. when the players take actions simultaneously). For the sake of simplicity, we will use "+" (respectively "-") to indicate positive (respectively. negative) utility function (payoff), and by 0 to indicate idle situation.

Table 1. Outcome of the green energy production game

\begin{tabular}{lll}
\hline Provider/Client & Consumes & Idle \\
\hline Produces & {$[+;+]$} & {$[-; 0]$} \\
Idle & {$[0 ;-]$} & {$[0 ; 0]$} \\
\hline
\end{tabular}

According to Table 1, one can observe the following:

- When energy is produced and consumed we have the first situation and both players can win,

- When energy is not produced but the consumer needs power, we consider that the client suffers a loss,

- When energy is produced but not consumed we consider that the provider loses money,

- When both players are idle

By applying the definition of a Nash Equilibrium (see [13] for more details) on Table 1, we can deduce that this game admits a unique Nash equilibrium: it corresponds to the outcome $([+;+])$.

For this kind of game, we can propose several strategies. We have restricted the study and the solution to the following strategies:

For the producer, the strategy is to find the best price, 
For the client, the strategy is to find the best amount of energy consumption according to the proposed price,

We can also adopt a reasoning similar to [1] with the global satisfaction of achieving an action in favor to humanity (subjective measures that are arbitrary) but we prefer to find an objective with simple measures to attract both players.

The utility function that we propose gives satisfaction to a consumer when he believes that green energy has reduced the energy cost. This is translated to a saving cost for this consumer.

We assume that the price dynamic. The same reasoning is applied to the energy operator as it saves expensive energy and hence reduces its cost.

We define $C_{c}(x, y)$ and $C_{\text {oper }}(x, y)$ to be the utility and revenue functions for consumer and operator respectively, where $x$ denotes the amount energy consumption chosen by the client and $y$, the price set by the operator.

The consumer will consume energy from the operator up to a certain limit that corresponds to his requirements as shown in Fig. 4.

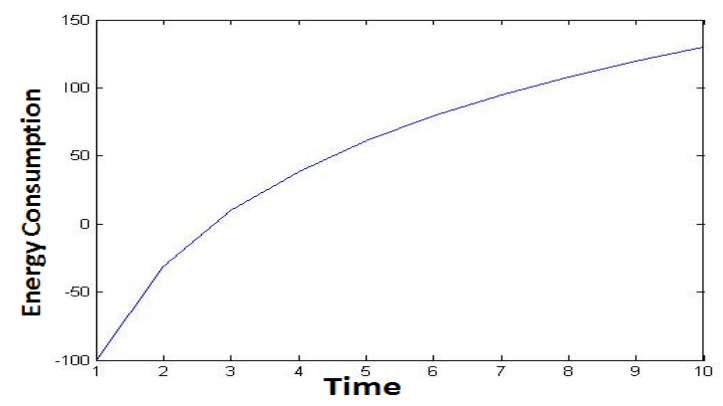

Fig. 4. Consumption pattern of the consumer

We now propose a mathematical formulation of the utility function for a consumer as a function of his consumption, assuming a fixed price by the operator, as follows:

$$
C_{c}(x, y)=A \ln (x)-y x
$$

where $\mathrm{A}$ is a positive constant $(\mathrm{A}>0), x$ is the consumption variable and $y$ is the price published by the operator.

Note that the normal consumption as shown in Figure 4 corresponds to a logarithmic progression but it is negatively affected if the price is increased. This kind of curves can be observed when charging an electric vehicle or with a normal domestic behavior.

We assume a typical revenue function of the operator given as follows:

$$
C_{\text {oper }}(x, y)=y x-\alpha y^{2}
$$

In the second term of (2), parameter $\alpha(\alpha>0)$ is used to regulate power when the price is very low, dissuading hence greedy consumption from a single client.

To deduce the optimal pricing strategy of the energy operator, and then finding the best consumption demand of a customer, we derive the following operations:

$$
\frac{\delta C_{\text {oper }}}{\delta y}=x-2 \alpha y=0 \Rightarrow x=2 a y
$$

In the following, we find the optimum response function for the consumer utility (see Fig. 5) by deriving with respect to cost. We first use (1) to replace the value of $x$ find in (3) in the client utility function (1). We obtain: 


$$
C_{c}(x, y)=A \ln (2 \alpha y)-2 \alpha y^{2}
$$

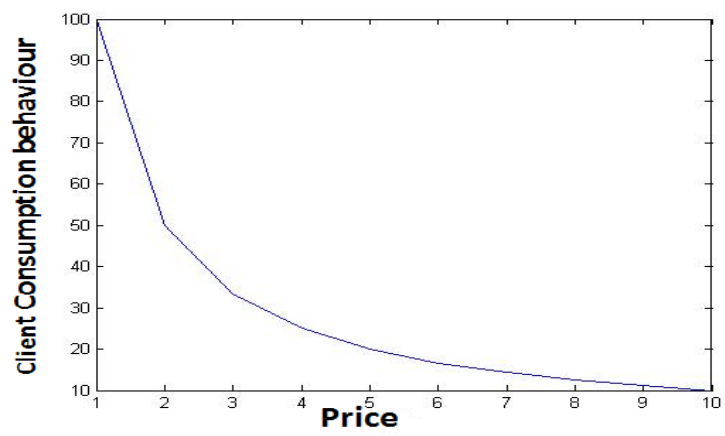

Fig. 5. Best response function for the consumer.

We seek for the maximum value of $C_{c}(x, y)$ as follows:

$$
\frac{\delta C_{c}}{\delta y}=\frac{A}{y}-4 \alpha y=0 \frac{-b \pm \sqrt{b^{2}-4 a c}}{2 a} \Rightarrow 4 \alpha y^{2}=A
$$

Which leads to deduce $\quad y=\frac{1}{2} \sqrt{\frac{A}{\alpha}}$.

Now we replace this value in (3) and we get easily $=\alpha \sqrt{\frac{A}{\alpha}}$.

Then the couple of strategies at the Nash equilibrium is given by:

$$
\left(x^{*} ; y^{*}\right)=\left(\alpha \sqrt{\frac{A}{\alpha}} ; \frac{1}{2} \sqrt{\frac{A}{\alpha}}\right)
$$

To prove the uniqueness of the Nash Equilibrium Point $\left(x^{*} ; y^{*}\right)$, we verify the following:

$$
\frac{\partial^{2} C_{c}}{\partial y^{2}}=-\frac{A}{y^{2}}-4 \alpha<0 \text { as } \alpha>0 \text { and } A>0
$$

Moreover, we also verify:

$$
\frac{\partial^{2} C_{o p e r}}{\partial y^{2}}=-2 \alpha<0 \text { as } \alpha>0
$$

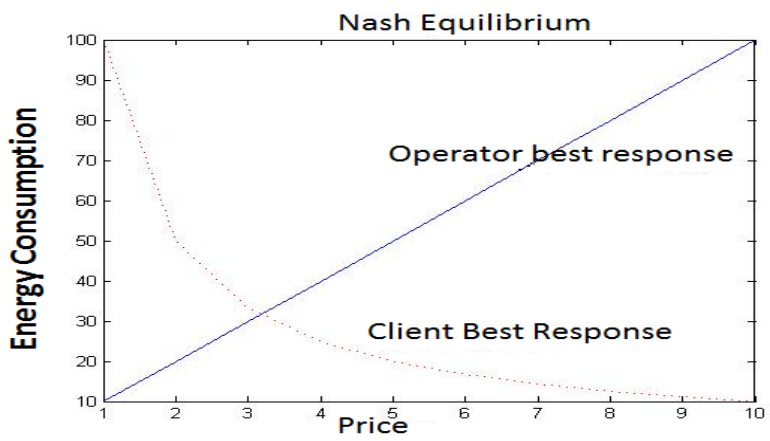

Fig. 6. Finding the nash equilibrium. 
This leads to conclude the uniqueness of the NEP found in (4). Fig. 6 shows clearly this result and the uniqueness of the NEP.

\section{Convergence to a Nash Equilibrium Point}

In this particular problem and with our assumptions, the two defined curves (operator and consumer payoffs) will intersect in a single point: the Nash equilibrium of the strategic game. Moreover, our simulations with MATLAB [19] illustrate that the strategies adopted by the two players (best reply dynamics) leads to the same result, and hence the system converges to this point after a certain number of iterations (see Fig. 7).

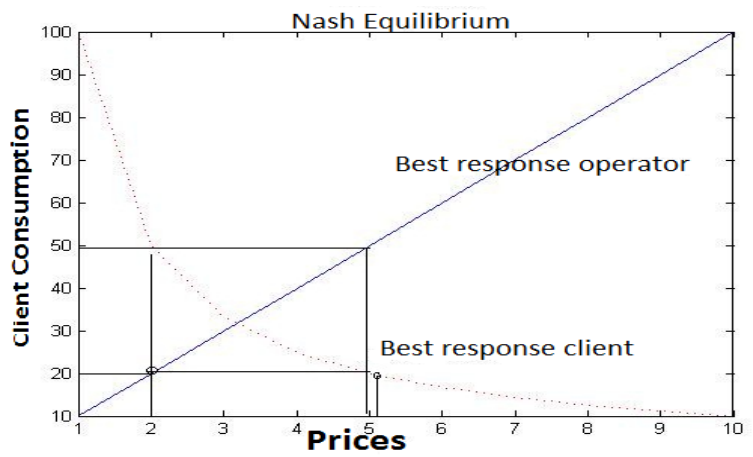

Fig. 7. Algorithm steps convergence towards equilibrium.

\subsection{Game assumptions}

By assuming that both players know all the characteristics of the game (both utility functions), and all players actions are known at each step of the strategic interaction, we precise the nature of a complete information game. As it is shown in Fig. 7, the algorithm in the operator side starts by assigning a price to the green power. The price here is high hence the client adopts certain consumption. Afterwards, the operator reacts to this and decreases price leading to increasing client consumption. This procedure is continued and converges to the equilibrium point.

\subsection{Analysis and potential extensions}

The resulting MATLAB application is based on the equations derived before but is enriched with many watchdogs that prevent for example from proposing too high or too low prices. We also need to adjust parameters to the kind and category of power meters. Details such as the nominal power, the presence of day/night tariffs, etc., are taken into account.

We note that the presented solution is suitable only for the first case in Table 1. We can extrapolate the system to propose a solution especially to encourage the client to consume green energy if he did not yet start any activity.

To enhance the quality of our approach, the equations (1) and (2) can be improved with several details to sketch better the kind of client or operator we are dealing with. Finally, one can adapt dynamic games where one of the players or both change his strategy during the game. The result should be different and depends on the new Nash equilibrium with under time constraints.

\section{Capturing the Cumulative Domestic Consumption}

This section describes a more realistic scenario that includes hundreds, or thousands of clients (consumers). In real life, consumers do not start or synchronize their energy consumption. Moreover, consumption peaks happen during known periods such as between 19 PM and 20 PM in Western Europe. 
This scenario can be modeled as shown in Fig. 8, in which we distinguish two levels: the operator and consumers levels.

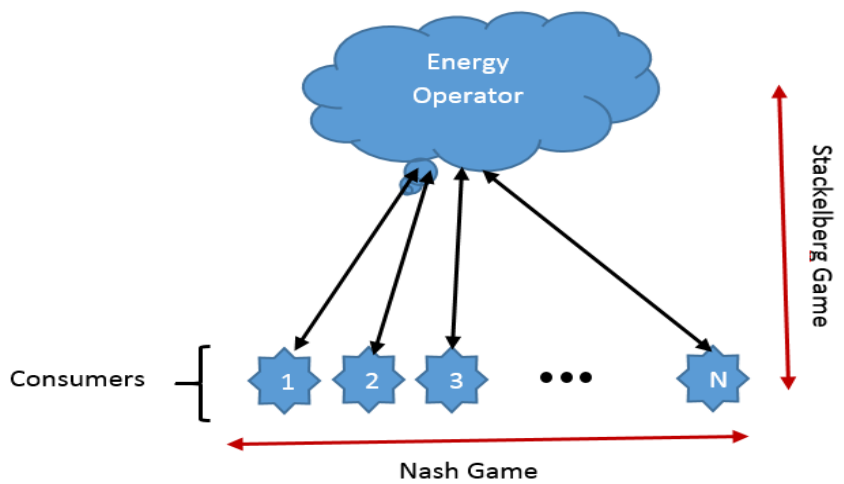

Fig. 8. One leader and multiple followers' game.

We note two cases for the pricing policy: uniform prices (all the considered customers have the same price), and differentiated prices (energy operator proposes different prices to consumers according to some criteria). In case of differentiated prices (respectively. uniform prices), we note by $y_{i}^{\text {oper }}$ (respectively. $y^{\text {oper }}$ ) the proposed price by the operator "oper" to consumer " $i$ ".

\subsection{Uniform prices cases}

In this case, we suppose that the operator suggests uniform prices to customers, so that $y_{i}^{\text {oper }}=y^{\text {oper }}$. Furthermore, normally, we would also add the time dimension in such a summation as customers start their electric devices in a desynchronized manner. However, as the consumption function is taking the dynamicity of cost as a variable, this can implicitly replace the effect of time and can hence represent the same effect of de-correlation between clients.

The game is now equivalent to the scenario where a consumer has the following objective function:

$$
C_{i}(x, y)=A_{i} \ln \left(x_{i}\right)-y^{\text {oper }} x_{i}
$$

To maximize the function (5), we give its derivative according to $x_{i}$, and let it equal to zero:

$$
\frac{\partial C_{i}(x, y)}{\partial x_{i}}=\frac{A_{i}}{x_{i}}-y^{\text {oper }}=0
$$

Solving equation ( $\backslash$ ref $\{$ derive 10$\}$ ) leads to obtain the following relation:

$$
x_{i}=\frac{A_{i}}{y^{\text {oper }}}
$$

In the other hand, the objective function of the operator is defined as follows:

$$
C_{\text {oper }}(x, y)=\sum_{i=1}^{N} y^{\text {oper }} x_{i}-\alpha_{i}\left(y^{\text {oper }}\right)^{2}
$$

Subject to limited resource constraints noted by “AR" for Available Resources within the operator:

$$
\sum_{i=1}^{N} x_{i} \leq A R
$$

To maximize the function (8) over the vector of demands $x$ and subject to the constraints given by (9), we use the Lagrangian method:

$$
L(y, \lambda)=\sum_{i=1}^{N} y^{\text {oper }} x_{i}-\alpha_{i}\left(y^{\text {oper }}\right)^{2}-\lambda\left[\sum_{i=1}^{N} x_{i}-A R\right]
$$


$\lambda$ is the Lagrangian multiplier. Let us now calculate the derivative of $L$ with respect to $y^{\text {oper }}$, and let it equal to zero:

$\frac{\partial L(y, \lambda)}{\partial y^{\text {oper }}}=\sum_{i=1}^{N} x_{i}-2 \alpha_{i} y^{\text {oper }}=0$

In the same manner, we also calculate the derivative of $L$ with respect to $\lambda$ :

$\frac{\partial L(y, \lambda)}{\partial \lambda}=\sum_{i=1}^{N} x_{i}-A R=0$

From (10), we deduce:

$$
\sum_{i=1}^{N} x_{i}=\sum_{i=1}^{N} 2 \alpha_{i} y^{\text {oper }}=2 y^{\text {oper }} \sum_{i=1}^{N} \alpha_{i}=2 y^{\text {oper }} N \bar{\alpha}
$$

where $\bar{\alpha}=\frac{1}{N} \sum_{i=1}^{N} \alpha_{i}$.

By using (11), we deduce:

$$
2 N \bar{\alpha} y^{\text {oper }}=A R
$$

Which leads to:

$$
\left(y^{\text {oper }}\right)^{*}=\frac{A R}{2 N \bar{\alpha}}
$$

From (7), we also get the optimal consumption of a client $\mathrm{i}$ :

$$
x_{i}^{*}=2 N \bar{\alpha} \frac{A_{i}}{A R}
$$

We deduce the Nash equilibrium point in case of uniform prices given by:

$$
\left(x_{i}^{*} ;\left(y^{\text {oper }}\right)^{*}\right)=\left(2 N \bar{\alpha} \frac{A_{i}}{A R} ; \frac{A R}{2 N \bar{\alpha}}\right)
$$

\subsection{Differentiated prices case}

We consider different possible prices proposed by the operator to different consumers. We assume that the prices are different from a geographic location to another, and the operator wants to favorite certain categories for different reasons. The net utility function of a consumer i is then given as follows:

$$
C_{i}\left(x_{i}, y\right)=A_{i} \ln \left(x_{i}\right)-y_{i}^{\text {oper }} x_{i}
$$

In the same manner, we give the revenue of the operator when dealing with $N$ customers:

$$
C_{\text {oper }}(x, y)=\sum_{i=1}^{N}\left(y_{i}^{\text {oper }} x_{i}-\alpha_{i}\left(y_{i}^{\text {oper }}\right)^{2}\right)
$$

Subject to resource available constraints:

$$
\sum_{i=1}^{N} x_{i} \leq A R
$$

We first maximize (12) to obtain the first relation between customer's consumption and the pricing suggested by the operator: 


$$
\frac{\partial C_{i}(x, y)}{\partial x_{i}}=\frac{A_{i}}{x_{i}}-y_{i}^{o p e r}=0
$$

This leads to obtain:

$$
x_{i}=\frac{A_{i}}{y_{i}^{\text {oper }}}, y_{i}^{\text {oper }}>0
$$

To maximize the function (13) over the vector of prices $y^{\text {oper }}$ and subject to the constraint given by (14), we use the Lagrangian method.

$$
L(y, \lambda)=\sum_{i=1}^{N}\left(y_{i}^{\text {oper }} x_{i}-\alpha_{i}\left(y^{\text {oper }}\right)^{2}\right)-\lambda\left[\sum_{i=1}^{N} x_{i}-A R\right]
$$

$\lambda$ is the Lagrangian multiplier. Let us now calculate the derivative form of $L$ with respect to $y_{i}^{\text {oper }}$, and let it equal to zero:

$$
\frac{\partial L(y, \lambda)}{\partial y_{i}^{\text {oper }}}=0
$$

In the same manner, we also calculate the derivative form of $L$ with respect to $\lambda$ :

$$
\frac{\partial L(y, \lambda)}{\partial \lambda}=0
$$

Solving the equation system composed by (16) and (17) leads to get:

$$
\sum_{i=1}^{N} x_{i}=\sum_{i=1}^{N} 2 \alpha_{i} y^{\text {oper }}=A R
$$

In which we deduce:

$$
\sum_{i=1}^{N} \alpha_{i} y^{\text {oper }}=A R / 2
$$

We now extract the $i^{t h}$ term from (18), and we get:

$$
\alpha_{i} y_{i}^{\text {oper }}+\sum_{u=1, u \neq i}^{N} \alpha_{u} y_{u}^{\text {oper }}=\frac{A R}{2}
$$

By summing (19) over all customers $i=1, \ldots, N$, we obtain:

$$
\sum_{i=1}^{N} \alpha_{i} y^{\text {oper }}+\sum_{i=1}^{N} \sum_{u=1, u \neq i}^{N} \alpha_{u} y_{u}^{\text {oper }}=\sum_{i=1}^{N} \frac{A R}{2}=\frac{A R}{2} N
$$

Which is equivalent to write:

$$
\frac{A R}{2}+N\left(\sum_{u=1, u \neq i}^{N} \alpha_{u} y_{u}^{o p e r}\right)=\frac{A R}{2} N
$$

this is also equivalent to:

$$
N\left(\sum_{u=1, u \neq i}^{N} \alpha_{u} y_{u}^{o p e r}\right)=\frac{A R}{2}(N-1)
$$

this leads to deduce:

$$
\left(\sum_{u=1, u \neq i}^{N} \alpha_{u} y_{u}^{\text {oper }}\right)=\frac{N-1}{N} \frac{A R}{2}=\left(1-\frac{1}{N}\right) \frac{A R}{2}
$$


then, (19) can be rewritten as follows:

$$
\alpha_{i} y_{i}^{o p e r}+\left(1-\frac{1}{N}\right) \frac{A R}{2}=\frac{A R}{2}
$$

it is not difficult to conclude that:

$$
\alpha_{i} y_{i}^{\text {oper }}=\frac{A R}{2 N}
$$

Thus, we can see that we reach similar result for the optimal consumer price:

$$
\left(y_{i}^{\text {oper }}\right)^{*}=\frac{A R}{2 N \alpha_{i}}
$$

and for an optimal consumption or energy demand of a consumer i:

$$
\left(x_{i}\right)^{*}=\frac{2 A_{i} \alpha_{i} N}{A R}
$$

The Nash Equilibrium Point in case of dynamic price and multiple clients is given by the couple:

$$
\left(\left(x_{i}\right)^{*} ;\left(y_{i}^{\text {oper }}\right)^{*}\right)=\left(\frac{2 A_{i} \alpha_{i} N}{A R} ; \frac{A R}{2 N \alpha_{i}}\right)
$$

\section{Summary and Future Work}

The Smart Grid context introduces a large number of interesting problems and optimizations to solve. Assuming the presence of a network infrastructure, precise real-time metering devices and prediction systems we have presented a game theory approach to encourage end customers to consume green energy as it is produced. The algorithm is based on the presence of two players: the operator and the customer and introduces a dynamic pricing strategy. The objective of the players is to reduce the energy cost and to have some fairness in green energy distribution. We show that such a system can have a Nash equilibrium point. A simple algorithm is described to reach this point.

We want to extend this work to more complex games with more actors, e.g. multiple providers scenarios where the consumer is also playing the operator role (e.g. it sails his too much produced renewable energy to his neighbors). We also plan to integrate this work into the prediction tools and distributed metering simulators we have already developed. These extension works will in particular be conducted in the context of SEAS ITEA3 project [5].

\section{Acknowledgements}

This research work has been carried out in the framework of the project Smart City Energy Analytics at Technological Research Institute SystemX, and therefore granted with public funds within the scope of the French Program "Investissements d'Avenir".

\section{References}

[1] VELCRI-Project. Financed by the National Energy Agency ADEME. (2010). [Online]. Available: http://www2.ademe.fr/servlet/doc?id=75098n\&view=standard

[2] Tushar W, Yuen C, Chai B, Smith DB, Poor H. Feasibility of using discriminate pricing schemes for energy trading in smart grid. In: Proc. Global Communications Conference, Dec. 2014:3138-3144.

[3] Taft J. The intelligent power grid-The energy and utilities project. Innovating for Transformation, 2006:74-76.

[4] Venayagamoorthy GK. Potentials and promises of computational intelligence for smart grids. In: Proc. IEEE Power and Energy Society, Calgary, Canada, 2009.

[5] ITEA3. Smart Energy Aware Systems. (2014). Project led by gdf-suez and started in February 2014 for a 3 years duration. 
[Online]. Available: https://itea3.org/project/seas.html

[6] Jose AA, Pahwa A. Economic evaluation of small wind generation ownership under different electricity pricing scenarios. In: Proc. of IEEE NAPS, 2010.

[7] Wang J, Biviji MA, Wang W. Lessons learned from smart grid enabled pricing programs. In: Proc. IEEE PECi, 2011:1-7.

[8] Faruqui A, Hledik R. The impact of dynamic pricing on westar energy. Smart Grid and Energy Storage Roundtable, Topeka, Kansas, 2009.

[9] Spees K, Lave L. Impacts of responsive load in pjm: load shifting and real time pricing. IAEE The Energy Journal, 2008:101112.

[10] Ibars C, Navarro M, Giupponi L. Distributed demand management in smart grid with a congestion game. In: Proc. IEEE Smart Grid Comm., 2010.

[11] Mohsenian-Rad A, Wong V, Jatskevich J, Schober J. Autonomous demand-side management based on game-theoretic energy consumption scheduling for the future smart grid. IEEE Transaction on Smart Grid, 2010:320-331.

[12] Bu S, Yu FR, Liu PX. Dynamic pricing for demand side management in the smart grid. In Proc. IEEE/ACM Green Com., 2011:47-51.

[13] Fudenberg D, Tirrol J. Game theory. In: Cambridge, MA: MIT Press; 1991.

[14] Ormond JM. Utility-based intelligent network selection in beyond 3g systems. In: Proc. IEEE ICC, 2006.

[15] Cocchi R, Shenker S, Estrin D, Zhang L. Pricing in computer networks: motivation, formulation, and example. IEEE/ACM Transactions on Networking, Dec. 1993; 1(6):614-627.

[16] Maggi L, Avrachenkov K, Cottatellucci L. Stochastic games for cooperative network routing and epidemic spread. In: Proc. ICC 2011 Workshop on Game Theory and Resource Allocation for 4G, 2011:1-5.

[17] TRAN PN. Modeles de decision pour la sélection d'interface et l'association flux/interface pour les terminaux mobiles multi interfaces. 2010.

[18] Daniel. (2014). Unpredictable wind energy - The danish dilemma. [Online]. Available: http://wilfriedheck.tripod.com/danish.htm

[19] Matlab. (2015). [Online]. Available: http://fr.mathworks.com/ 